\title{
A study on normal reference values of echocardiographic chamber dimensions in new born infants from eastern India- a pilot study
}

\author{
Swapan Halder ${ }^{1}$, Samir Roy ${ }^{1}$, Anindya Mukherjee ${ }^{2}$, Dibbendhu Khanra ${ }^{3}$, Soumyajit Saha ${ }^{1}$, \\ and Manoranjan Mandal ${ }^{1}$ \\ ${ }^{1}$ Affiliation not available \\ ${ }^{2}$ NRS Medical College \\ ${ }^{3}$ Royal Wolverhampton Hospitals NHS Trust
}

May 2, 2021

\begin{abstract}
Introduction: Echocardiography has been an integral part of neonatal care since the 1990s and application of this technology is increasing ever since. It is already known that differences in weight, age and race significantly affect the normal heart dimensions. Therefore, establishing the normative values for cardiac dimensions among Indian healthy newborn infants is of paramount importance. In this study we report the normal reference values of echocardiographic chamber dimensions of newborn infants from eastern India. Methods: 100 healthy newborn infants were included in this cross-sectional, observational, single hospital-based pilot study. A single experienced cardiologist performed the trans-thoracic echocardiographic studies on the subjects in supine position in their respective bassinets. No sedation was used. The parameters were distributed according to body weight and in percentiles. Results: Subjects were categorized according to their body weight into three groups: $2-2.4 \mathrm{~kg}$, 2.5-2.9 kg and 3-3.5 kg. Each group comprises of 7, 50 and 43 newborns respectively. Left ventricular end-diastolic dimension and left ventricular end-systolic dimension showed incremental trends across body weight. Conclusion: This study shows the difference of cardiac dimensions in Indian newborn infants from western data. This is the first study presenting the normative reference ranges and percentiles of echocardiographic parameters in Indian newborn infants which needs confirmation with larger multicenter studies.
\end{abstract}

TITLE PAGE

Manuscript type: Original article

Title : A study on normal reference values of echocardiographic chamber dimensions in new born infants from eastern India- a pilot study

Running Title: Echocardiographic parameters in neonates

Authors

Swapan Kumar Halder, MD, DM

Department of Cardiology, NRS Medical College, Kolkata, India

drskh@rediffmail.com

Samir Roy, MD, DM

Department of Cardiology, NRS Medical College, Kolkata, India

Skrr80@yahoo.in 
Anindya Mukherjee, MD, DM

Department of Cardiology, NRS Medical College, Kolkata, India

anindya768@yahoo.co.in

Dibbendhu Khanra, MD, DM

Electrophysiology and Devices, Royal Wolverhampton NHS Trust, UK

ddk3987@gmail.com

Soumyojit Saha, MD, DM

Department of Cardiology, Narayana Multispeciality Hospital, Howrah, India

asit768@gmail.com

Manoranjan Mandal

Department of Cardiology, NRS Medical College, Kolkata, India

drmandal6162@gmail.com

\section{Corresponding Author:}

Anindya Mukherjee, MD Medicine, DM Cardiology

Senior Resident, Department of Cardiology, NRS Medical College, Kolkata, India

Visiting Cardiologist, Department of Cardiology, AMRI Hospitals, Kolkata, India

Visiting Cardiologist, Department of Cardiology, Ruby General Hospital, Kolkata, India

Postal Address: Department of Cardiology, NRS Medical College, 138, A.J.C. Bose Road, Kolkata 700014, India

Phone: +919073102366

Email id: anindya768@yahoo.co.in

Contributions: SKH, SR, SS: Concept and design, acquisition of data and interpretation, critical review and final approval of the study; AM, DK: Concept and design, interpretation of data, drafting the article, critical review and final approval of the study; MM: Concept and design, critical review and final approval of the study.

Acknowledgements: None

Conflict of interest: None

Role of Funding Source: None

Word Count of the Abstract: 210

Word Count of the Text: 2318

Number of Tables: 4

Number of Figures: 2

The ethical standards of the responsible committee on human experimentation (institutional and national) and the Helsinki Declaration of 1964 and later revisions were followed in all steps of research. Parents of all the subjects gave informed consent for the study. Institutional Review Board approved the study.

Title : A study on normal reference values of echocardiographic chamber dimensions in new born infants from eastern India- a pilot study 
Running Title: Echocardiographic parameters in neonates

\begin{abstract}
Introduction: Echocardiography has been an integral part of neonatal care since the 1990s and application of this technology is increasing ever since. It is already known that differences in weight, age and race significantly affect the normal heart dimensions. Therefore, establishing the normative values for cardiac dimensions among Indian healthy newborn infants is of paramount importance. In this study we report the normal reference values of echocardiographic chamber dimensions of newborn infants from eastern India.
\end{abstract}

Methods: 100 healthy newborn infants were included in this cross-sectional, observational, single hospitalbased pilot study. A single experienced cardiologist performed the trans-thoracic echocardiographic studies on the subjects in supine position in their respective bassinets. No sedation was used. The parameters were distributed according to body weight and in percentiles.

Results: Subjects were categorized according to their body weight into three groups: $2-2.4 \mathrm{~kg}, 2.5-2.9 \mathrm{~kg}$ and 3-3.5 kg. Each group comprises of 7,50 and 43 newborns respectively. Left ventricular end-diastolic dimension and left ventricular end-systolic dimension showed incremental trends across body weight.

Conclusion: This study shows the difference of cardiac dimensions in Indian newborn infants from western data. This is the first study presenting the normative reference ranges and percentiles of echocardiographic parameters in Indian newborn infants which needs confirmation with larger multicenter studies.

Keywords: Echocardiography, Newborn infants, Percentiles, Pilot study, Reference range

\title{
Introduction :
}

Echocardiography has been an integral part of neonatal care since the 1990s and application of this technology is increasing ever since $[1,2,3]$. "Functional", "point of care", "target" or "focus" echocardiography has become an invaluable tool in neonatal and pediatric intensive care setting [4]. The concept of "neonatologistperformed echocardiography" (NPE) has developed for improved cardiorespiratory care of newborn [5]. Several small studies have suggested normative data related to cardiac chambers in infants and newborn over last 50 years but none has been reported from the Indian subcontinent and no clear guidelines exist unlike adults [6]. A prior study by Mukherjee et al showed that echocardiographic chamber dimensions among normal Indian subjects not only varied from the western data but also varied among different parts of the country [7]. It is already known that differences in weight, age and race significantly affect the normal heart dimensions [8-10]. Therefore, establishing the normative values for cardiac dimensions among Indian healthy newborn infants is of paramount importance. In this study we report the normal reference values of echocardiographic chamber dimensions of newborn infants from eastern India.

\section{Methodology:}

Subject recruitment

100 healthy newborn infants (from 24 hours after birth up to seven days) delivered at term by vaginal delivery or caesarean section from February 2020 to January 2021, were included in this cross-sectional, observational, single hospital-based pilot study. Subject recruitment process has been depicted in Figure 1. The ethical standards of the responsible committee on human experimentation (institutional and national) and the Helsinki Declaration of 1964 and later revisions were followed in all steps of research. Parents of all the subjects gave informed consent for the study. Institutional Review Board approved the study.

Echocardiography

A single experienced cardiologist performed the trans-thoracic echocardiographic studies using a Vivid S5 machine with a M7S matrix array probe with a frequency range from 5.7 to $7.2 \mathrm{MHz}$ (GE Vingmed, Horten, Norway). Machine integrated ECG recording was performed simultaneously. Each measurement was taken three times and averaged [6]. 
The echocardiography was performed on the subject in supine position in his/her respective bassinet $[6,11]$. No sedation was used although a pacifier nipple was sometimes required to keep the neonate quiet and at rest $[6,11]$.

Left atrial anteroposterior diameters (LAd) were measured on parasternal long axis view at end systole $[5,11,12]$. Aortic root diameters (at maximal diameter of sinus of Valsalva) (Ao) were measured from the same view. 2D guided parasternal short axis view was used to measure thickness of the interventricular septum at end diastole (IVSd), left ventricular dimension at end diastole (LVEdD), left ventricular dimension at end systole (LVEsD) and left ventricular posterior wall thickness at end diastole (LVPWd) from leading edge of the septal endocardium to the leading edge of posterior wall endocardium as per the recent guidelines $[5,11$ 13]. Similar leading edge to leading edge convention was used for LA and right ventricular outflow dimension in end-diastole (RVEdD) measurement. Right ventricular anterior wall thickness at end diastole (RVAWd) and RVEdD at tricuspid annulus were measured in 2-D apical 4-chamber view [12]. Main pulmonary artery diameter at the level of semilunar valve (PA) was measured from parasternal short axis view in systole [12]. LV ejection fraction (LVEF) and fractional shortening (LVFS) were measured using the biplane Simpson rule $[5,12]$.

Echocardiographic dimensions were evaluated using body weight since body surface area changed minimally in this population [6].

\section{Statistical analysis}

The mean \pm 2 standard deviation (SD) rule was followed to propose normal echocardiographic reference values in this study. This is based on the assumption that $95 \%$ of values of a reference group is in this range, and the sample value can be greater than upper limit or lesser than lower limit in $2.5 \%$ of the time respectively, whatever might be the distribution of these values [14].

For statistical analysis data were analyzed by SPSS (version 24.0; SPSS Inc., Chicago, IL, USA) and GraphPad Prism version 5. Summarization of data was done as mean and standard deviation for numerical variables and count and percentages for categorical variables. Percentile wise distribution of data was performed.

\section{Results:}

Subjects were categorized according to their body weight into three groups: 2-2.4 kg, 2.5-2.9 kg and 3-3.5 $\mathrm{kg}$. Each group comprises of 7, 50 and 43 newborns respectively.

Means of chamber dimensions

Mean \pm standard deviation (SD) values of each of eleven cardiac dimensions as mentioned above for newborns in relation to body weight were calculated as shown in Table 1. Parameters except mean LVEdD and LVEsD did not show any incremental trend with increase of body weight.

Normal reference range

Normal reference ranges of echocardiographic chamber dimensions are depicted in Table 2. As evident from the mean values, only LVEdD and LVEsD showed incremental values of upper normal reference limit and lower normal reference limit with increase of body weight. Other parameters did not show any clear trend with increasing body weight.

Percentiles of the values of echocardiographic measurements

Percentile value (5th, 10th, 25th, 50th, 75th, 90th, and 95th centiles) for each of the cardiac dimensions were calculated and shown in Table 3.

Distribution of echocardiographic parameters according to body weight

Figure 2 is a composite of scatterplots showing the spread of the various echocardiographic parameters across body weight. As evident from the earlier results, only the values of LVEdD and LVEsD shows some positive relationship with increasing body weight. 


\section{Discussion:}

Heterogeneity of echocardiographic parameters

The wide range of changes during normal development of the cardiac structures require a large study group for accurate data on normal values, even when centile charts are used $[15,16]$. Studies from different parts of the world have shown similarity as well as disparity of echocardiographic parameters among neonates $[6,15]$. In addition, a study conducted by DerSimonian and Levine showed that data obtained from multiple small population studies and combined by meta-analysis has a drawback in that they are overtly affected by the heterogeneity of the populations examined [17]. With the heterogeneity of data from different parts of the world in background, Bansal et al conducted a pilot study to assess the echocardiographic parameters in northern Indian adults [18]. A recent study from our centre confirmed the regional heterogenicity of echocardiographic parameters in young adults [7]. Therefore, the need for regional references of the basic echocardiographic parameters in newborn infants is paramount. To the best of our knowledge, there are no studies that evaluated healthy term Indian neonates to develop centile charts.

Gender variation

Gender appeared only a significant determinant for LVEdD among children in a Dutch study [19]. If gender would have been an important determinant, it would have been significant for all cardiac dimensions, since the expectation has to be that a larger heart needs thicker walls because of Laplace's law. So, if boys would have larger LV dimensions, it would be expected that the walls are thicker as well, which was not observed. Therefore, it was chosen to establish new reference ranges of cardiac dimensions independent of gender. This is in accordance with the results of other studies $[6,11,15,20,21]$.

Studies on echocardiographic parameters of newborn infants

Only a handful number of studies from different parts of the world report normative reference ranges of echocardiographic parameters in sizeable population of newborn infants $[6,11,15,22]$. The studies by Kampmann et al and Güzeltaş et al followed the old American Society of Echocardiography recommendations in measuring the echocardiographic parameters whereas our study followed methods in measuring the chamber dimensions in accordance with the current guidelines [5,12].

Echocardiographic dimensions and body weight

Güzeltaş et al found that right and left ventricular, left atrial and aortic dimensions increased linearly with body weight while in our study only LVEdD and LVEsD showed incremental trends with body weight [6].

Comparison of echocardiographic parameters across different studies

Table 4 shows normal echocardiographic values in studies that include 100 or more healthy newborn infants. RVEdD, LVEdD and LVEsD were lesser than the earlier studies which can be attributed to interobserver variability, regional and ethnic differences $[6,11,15,22]$. Moreover, with time the temporal and spatial resolution of echocardiographic scanners have improved yielding more exact measurements [6]. Unlike in this study and that by Güzeltaş et al where a single cardiologist performed all the procedures, measurements were recorded by several cardiologists in the earlier studies [6,11,15,22]. Lad and Ao had similar range of measurement in our study and that by Güzeltas et al, Kampmann et al but lower than those by Solinger et al and Hagan et al $[6,11,15,22]$. This difference can be due to the reasons already stated. The fact that the earlier studies were conducted even before the American Society of Echocardiography recommendations came into place should be taken into account $[11,22,23]$. For example, exterior dimension of the aorta was measured in the study by Solinger et al [22]. LVEF found in our study was similar to the study by Güzeltaş et al [6].

\section{Limitations:}

Omission of measurement of detailed right ventricular dimensions and function was a major drawback. Doppler parameters were not included in the study. This was a pilot study on only 100 newborn infants 
from a large volume tertiary care centre of eastern India and a larger sample size from various parts of the country is needed to confirm the findings. Like most studies, our study included body weights of $2-4.5 \mathrm{~kg}$, but among the study population no newborn infant was found to have body weight $>3.5 \mathrm{~kg}$. Hence direct comparison of parameters with other studies cannot be done. Lastly, interobserver variability remains a hindrance while comparing datasets from various parts of the world.

\section{Conclusion:}

This study which included newborn infants from eastern India reaffirms that Indian subjects have smaller cardiac chamber diameters compared to western population. This is the first study presenting the normative reference ranges and percentiles of echocardiographic parameters in Indian newborn infants which needs confirmation with larger multicenter studies. The normal reference ranges and percentiles of echocardiographic measurements will be of immense value while treating a sick newborn or preparing Indian neonatal echocardiographic guidelines. This study will pave the way for further research on echocardiography of Indian newborn infants.

\section{What is Already Known:}

Differences in weight, age and race significantly affect the normal heart dimensions.

\section{What This Study adds:}

This is the first study presenting the normative reference ranges and percentiles of echocardiographic parameters in Indian newborn infants.

\section{Reference}

1. Skinner JR. Echocardiography on the neonatal unit: a job for the neonatologist or the cardiologist? Arch. Dis. Child. 1998;78,:401-2.

2. El-Khuffash AF, McNamara PJ. Neonatologist-performed functional echocardiography in the neonatal intensive care unit. Semin. Fetal Neonatal Med. 2011;16:50-60.

3. Evans N, Gournay V, Cabanas F, et al. Point-of-care ultrasound in the neonatal intensive care unit: international perspectives. Semin Fetal Neonatal Med. 2011;16:61-8. doi: 10.1016/j.siny.2010.06.005.

4. Tissot C, Muehlethaler V, Sekarski N. Basics of Functional Echocardiography in Children and Neonates. Front Pediatr. 2017;5:235. doi: 10.3389/fped.2017.00235.

5. Mertens L, Seri I, Marek J, et al; Writing Group of the American Society of Echocardiography; European Association of Echocardiography; Association for European Pediatric Cardiologists. Targeted Neonatal Echocardiography in the Neonatal Intensive Care Unit: practice guidelines and recommendations for training. Writing Group of the American Society of Echocardiography (ASE) in collaboration with the European Association of Echocardiography (EAE) and the Association for European Pediatric Cardiologists (AEPC). J Am Soc Echocardiogr. 2011;24:1057-78. doi: 10.1016/j.echo.2011.07.014.

6. Güzeltaş A, Eroğlu AG. Reference values for echocardiographic measurements of healthy newborns. Cardiol Young. 2012;22:152-7. doi: 10.1017/S1047951111001259.

7. Mukherjee A, Halder SK, Nandi S, et al. A study on normal reference values of echocardiographic chamber dimensions in young eastern Indian adults. Indian Heart J. 2021;73:77-84. doi: 10.1016/j.ihj.2020.12.010.

8. Poppe KK, Doughty RN, Gardin JM, et al. Ethnic-specific normative reference values for echocardiographic LA and LV size, LV mass, and systolic function: the EchoNoRMAL study. JACC (J Am Coll Cardiol): Cardiovascular Imaging. 2015;8:656e665.

9. Pfaffenberger S, Bartko P, Graf A, et al. Size matters! Impact of age, sex, height, and weight on the normal heart size. CircCardiovasc Imaging. 2013;6:1073e1079.

10. Poppe KK, Bachmann ME, Triggs CM, et al. Geographic variation in left ventricular mass and mass index: a systematic review. J Hum Hypertens. 2012;26:420e429.

11. Hagan AD, Deely WJ, Sahn D, et al. Echocardiographic criteria for normal newborn infants. Circulation. 1973;48:1221-6. doi: 10.1161/01.cir.48.6.1221. 
12. Lopez L, Colan SD, Frommelt PC, et al. Recommendations for quantification methods during the performance of a pediatric echocardiogram: a report from the Pediatric Measurements Writing Group of the American Society of Echocardiography Pediatric and Congenital Heart Disease Council. J Am Soc Echocardiogr. 2010;23:465-95; quiz 576-7. doi: 10.1016/j.echo.2010.03.019.

13. Lang RM, Badano LP, Mor-Avi V, et al. Recommendations for cardiac chamber quantification by echocardiography in adults: an update from the American Society of Echocardiography and the European Association of Cardiovascular Imaging. J Am Soc Echocardiogr. 2015;28:1e39.

14. Marshal W, Bangert S. Clinical Biochemistry: Metabolic and Clinical aspects. Philadelphia: Churchill Livingstone/Elsevier; 2008:19.

15. Kampmann C, Wiethoff CM, Wenzel A, et al. Normal values of M mode echocardiographic measurements of more than 2000 healthy infants and children in central Europe. Heart. 2000;83:667-72

16. Epstein ML, Golberg SJ, Allen HD, et al. Great vessel, cardiac chamber and wall growth patterns in normal children. Circulation. 1975;51:124-9.

17. DerSimonian R, Levine RJ. Resolving discrepancies between a meta-analysis and a subsequent large controlled trial. JAMA. 1999;282:664-70.

18. Bansal M, Mohan JC, Sengupta SP. Normal echocardiographic measurements in Indian adults: how different are we from the western populations? A pilot study. Indian Heart J. 2016;68:772e775.

19. Overbeek L.I.H, Kapusta L, Peer P.G.M, et al. New reference values for echocardiographic dimensions of healthy Dutch children. Eur J Echocardiography. 2006;7:113-121.

20. Hanseus K, Bjo"rkhem G, Lundstro"m N-R. Dimensions of cardiac chambers and great vessels by cross-sectional echocardiography in infants and children. Pediatr Cardiol. 1988;9:7-15.

21. Daubaney PEF, Blackstone EH, Weintraub RG, et al. Relationship of the dimension of cardiac structures to body size: an echocardiographic study in normal infants and children. Cardiol Young. 1999;9:40210 .

22. Solinger R, Elbl F, Minhas K. Echocardiography in the normal neonate. Circulation. 1973;47:108-18.

23. Sahn D, DeMaria A, Kisslo J, et al. The committee on M-mode standardization of the American Society of Echocardiography: recommendation regarding quantitation in M-mode echocardiography: results of a survey of echocardiographic methods. Circulation. 1978; 58: 1072-183.

Table 1: Mean values of echocardiographic chamber dimensions according to body weight in eastern Indian newborn infants

\begin{tabular}{llll}
\hline Parameters & $\begin{array}{l}\text { Body weight 2.0-2.4 kg } \\
(\mathrm{n}=7)\end{array}$ & $\begin{array}{l}\text { Body weight 2.5-2.9 kg } \\
(\mathrm{n}=50)\end{array}$ & $\begin{array}{l}\text { Body weight 3.0-3.5 kg } \\
(\mathrm{n}=43)\end{array}$ \\
\hline RVAWd & $3.64 \pm 0.57$ & $3.58 \pm 0.58$ & $3.13 \pm 0.70$ \\
RVEdD & $11.04 \pm 1.04$ & $11.12 \pm 1.25$ & $10.80 \pm 1.12$ \\
IVSd & $3.51 \pm 0.43$ & $3.59 \pm 0.37$ & $3.33 \pm 0.55$ \\
LVEdD & $13.06 \pm 1.24$ & $14.90 \pm 1.83$ & $15.37 \pm 1.29$ \\
LVEsD & $8.09 \pm 1.12$ & $9.53 \pm 1.75$ & $10.32 \pm 1.31$ \\
LVPWd & $2.98 \pm 0.13$ & $3.24 \pm 0.51$ & $3.06 \pm 0.49$ \\
PA & $8.15 \pm 0.57$ & $8.17 \pm 1.38$ & $8.02 \pm 1.35$ \\
Ao & $8.26 \pm 0.68$ & $8.36 \pm 1.13$ & $8.02 \pm 0.91$ \\
LAd & $9.50 \pm 0.76$ & $10.38 \pm 1.41$ & $9.89 \pm 1.58$ \\
LVFS & $38.00 \pm 6.17$ & $37.65 \pm 4.65$ & $36.40 \pm 2.82$ \\
LVEF & $71.63 \pm 7.97$ & $71.65 \pm 5.92$ & $70.63 \pm 3.99$ \\
\hline
\end{tabular}

Ao- Aortic root dimension, mm; IVSd- Septal thickness at end diastole, mm; LAd- Left atrial anteroposterior dimension, $\mathrm{mm}$; LVEdD-left ventricular end diastolic dimension, $\mathrm{mm}$; LVEF- left ventricular ejection fraction, $\%$; LVEsD-left ventricular end systolic dimension, mm; LVFS- left ventricular fractional shortening, \%; LVPWd-Posterior wall thickness at end diastole, mm; PA- main pulmonary artery diameter, mm; RVAWdRight ventricular anterior wall thickness at end diastole, mm; RVEdD- Right ventricle outflow diameter at 
end diastole, $\mathrm{mm}$.

Table 2: Normal reference ranges of echocardiographic chamber dimensions in eastern Indian newborn infants

\begin{tabular}{llll}
\hline Parameters & $\begin{array}{l}\text { Body weight 2.0-2.4 kg } \\
(\mathrm{n}=7)\end{array}$ & $\begin{array}{l}\text { Body weight 2.5-2.9 kg } \\
(\mathrm{n}=50)\end{array}$ & $\begin{array}{l}\text { Body weight 3.0-3.5 kg } \\
(\mathrm{n}=43)\end{array}$ \\
\hline RVAWd & $2.51-4.77$ & $2.43-4.74$ & $1.73-4.53$ \\
RVEdD & $8.96-13.11$ & $8.62-13.63$ & $8.49-13.11$ \\
IVSd & $2.65-4.37$ & $2.86-4.31$ & $2.24-4.42$ \\
LVEdD & $10.59-15.53$ & $11.24-18.57$ & $12.80-17.93$ \\
LVEsD & $5.85-10.32$ & $6.04-13.01$ & $7.70-12.94$ \\
LVPWd & $2.72-3.23$ & $2.22-4.27$ & $2.08-4.03$ \\
PA & $7.01-9.29$ & $5.42-10.92$ & $5.33-10.72$ \\
Ao & $6.91-9.61$ & $6.10-10.62$ & $6.21-9.82$ \\
LAd & $7.98-11.02$ & $7.56-13.19$ & $6.73-13.04$ \\
LVFS & $25.67-50.33$ & $28.35-46.96$ & $30.77-42.02$ \\
LVEF & $55.70-87.55$ & $59.82-83.49$ & $62.64-78.62$ \\
\hline
\end{tabular}

Ao- Aortic root dimension, mm; IVSd- Septal thickness at end diastole, mm; LAd- Left atrial anteroposterior dimension, mm; LVEdD-left ventricular end diastolic dimension, $\mathrm{mm}$; LVEF- left ventricular ejection fraction, $\%$; LVEsD-left ventricular end systolic dimension, mm; LVFS- left ventricular fractional shortening, \%; LVPWd-Posterior wall thickness at end diastole, mm; PA- main pulmonary artery diameter, mm; RVAWdRight ventricular anterior wall thickness at end diastole, mm; RVEdD- Right ventricle outflow diameter at end diastole, $\mathrm{mm}$.

Table 3: Percentiles of the values of echocardiographic measurements in eastern Indian newborn infants

\begin{tabular}{llllllll}
\hline & Percentiles & Percentiles & Percentiles & Percentiles & Percentiles & Percentiles & Percentiles \\
\hline & $\mathbf{5}$ & $\mathbf{1 0}$ & $\mathbf{2 5}$ & $\mathbf{5 0}$ & $\mathbf{7 5}$ & $\mathbf{9 0}$ & 95 \\
RVAWd & 2.21 & 2.41 & 3.00 & 3.40 & 3.80 & 4.20 & 4.49 \\
RVEdD & 9.00 & 9.20 & 9.85 & 11.20 & 11.98 & 12.40 & 12.70 \\
IVSd & 2.40 & 3.00 & 3.23 & 3.50 & 3.80 & 4.10 & 4.10 \\
LVEdD & 11.31 & 13.31 & 14.10 & 14.85 & 16.00 & 17.09 & 17.79 \\
LVEsD & 7.11 & 7.33 & 8.50 & 9.55 & 11.10 & 11.79 & 12.79 \\
LVPWd & 2.40 & 2.42 & 2.80 & 3.00 & 3.48 & 3.80 & 4.10 \\
PA & 5.82 & 6.40 & 7.20 & 8.00 & 9.10 & 9.40 & 9.69 \\
Ao & 6.50 & 7.10 & 7.35 & 8.20 & 9.00 & 9.30 & 10.29 \\
LA & 7.33 & 8.40 & 9.200 & 10.10 & 11.00 & 11.79 & 12.00 \\
LVFS & 31.00 & 32.00 & 35.00 & 37.00 & 39.00 & 42.00 & 45.00 \\
LVEF & 64.00 & 65.00 & 68.00 & 71.00 & 75.00 & 76.00 & 80.00 \\
\hline
\end{tabular}

Ao- Aortic root dimension, mm; IVSd- Septal thickness at end diastole, mm; LAd- Left atrial anteroposterior dimension, $\mathrm{mm}$; LVEdD-left ventricular end diastolic dimension, $\mathrm{mm}$; LVEF- left ventricular ejection fraction, $\%$; LVEsD-left ventricular end systolic dimension, mm; LVFS- left ventricular fractional shortening, \%; LVPWd-Posterior wall thickness at end diastole, mm; PA- main pulmonary artery diameter, mm; RVAWdRight ventricular anterior wall thickness at end diastole, mm; RVEdD- Right ventricle outflow diameter at end diastole, $\mathrm{mm}$.

Table 4: Normal echocardiographic values in studies that include 100 or more healthy newborns. 


\begin{tabular}{|c|c|c|c|c|c|c|c|}
\hline & $\begin{array}{l}\text { Our study } \\
(\mathrm{n}=100)\end{array}$ & $\begin{array}{l}\text { Our study } \\
(\mathrm{n}=100)\end{array}$ & $\begin{array}{l}\text { Our study } \\
(\mathrm{n}=100)\end{array}$ & $\begin{array}{l}\text { Güzeltaş } \\
\text { et al }^{6}(\mathrm{n}= \\
250)\end{array}$ & $\begin{array}{l}\text { Kampmann } \\
\text { et } \mathrm{al}^{15} \\
(\mathrm{n}=-)\end{array}$ & $\begin{array}{l}\text { Solinger et } \\
\mathrm{al}^{22}(\mathrm{n}= \\
240)\end{array}$ & $\begin{array}{l}\text { Hagan et } \\
\mathrm{al}^{11}(\mathrm{n}= \\
200)\end{array}$ \\
\hline Parameters & $\begin{array}{l}\text { Body weight } \\
2.0-2.4 \mathrm{~kg} \\
(\mathrm{n}=7)\end{array}$ & $\begin{array}{l}\text { Body weight } \\
2.5-2.9 \mathrm{~kg} \\
(\mathrm{n}=50)\end{array}$ & $\begin{array}{l}\text { Body weight } \\
3.0-3.5 \mathrm{~kg} \\
(\mathrm{n}=43)\end{array}$ & $\begin{array}{l}\text { Body weight } \\
2-4.5 \mathrm{~kg}\end{array}$ & $\begin{array}{l}\text { Body weight } \\
2-4.5 \mathrm{~kg}\end{array}$ & $\begin{array}{l}\text { Body weight } \\
2.27-4.54 \mathrm{~kg}\end{array}$ & $\begin{array}{l}\text { Body weight } \\
2.7-4.5 \mathrm{~kg}\end{array}$ \\
\hline RVAWd & $2.51-4.77$ & $2.43-4.74$ & $1.73-4.53$ & $1.3-4$ & $1.3-3.7$ & $1.1-4.1$ & $2-4.7$ \\
\hline RVEdD & 8.96- 13.11 & $8.62-13.63$ & 8.49- 13.11 & $3.3-12.2$ & $4-13.1$ & $10.4-17.7$ & $6.1-15$ \\
\hline IVSd & 2.65- 4.37 & 2.86- 4.31 & 2.24- 4.42 & $1.7-5.2$ & $2.1-5.2$ & $2.1-4.5$ & $1.8-4$ \\
\hline LVEdD & $10.59-15.53$ & $11.24-18.57$ & $12.80-17.93$ & $11.7-22.1$ & $15-23.3$ & $16.1-24.1$ & $12-23.3$ \\
\hline LVEsD & 5.85- 10.32 & 6.04- 13.01 & 7.70- 12.94 & $6.6-15.7$ & $9.7-15.2$ & $6.8-13.5$ & 8-18.6 \\
\hline LVPWd & $2.72-3.23$ & $2.22-4.27$ & $2.08-4.03$ & $1.6-5.2$ & $1.9-4.8$ & $2.1-4.5$ & $1.6-3.7$ \\
\hline $\mathrm{PA}$ & 7.01- 9.29 & $5.42-10.92$ & 5.33- 10.72 & - & - & - & - \\
\hline Ao & $6.91-9.61$ & $6.10-10.62$ & $6.21-9.82$ & $5.6-11.7$ & $6.9-11.6$ & $9.3-13.6$ & $8.1-12$ \\
\hline LAd & 7.98- 11.02 & 7.56- 13.19 & $6.73-13.04$ & $6.6-16.4$ & $8.3-16.9$ & $6.8-13.5$ & $5-10$ \\
\hline LVFS & 25.67- 50.33 & 28.35- 46.96 & $30.77-42.02$ & 19.9- 47.66 & - & - & - \\
\hline LVEF & $55.70-87.55$ & 59.82- 83.49 & 62.64- 78.62 & $50.02-89.22$ & - & - & - \\
\hline
\end{tabular}

Ao- Aortic root dimension, mm; IVSd- Septal thickness at end diastole, mm; LAd- Left atrial anteroposterior dimension, mm; LVEdD-left ventricular end diastolic dimension, $\mathrm{mm}$; LVEF- left ventricular ejection fraction, \%; LVEsD-left ventricular end systolic dimension, mm; LVFS- left ventricular fractional shortening, \%; LVPWd-Posterior wall thickness at end diastole, mm; PA- main pulmonary artery diameter, mm; RVAWdRight ventricular anterior wall thickness at end diastole, mm; RVEdD- Right ventricle outflow diameter at end diastole, $\mathrm{mm}$.

Figure legends

Figure 1. Flow chart showing subject recruitment pattern

Figure 2: Distribution of echocardiographic parameters according to body weight

Ao- Aortic root dimension, mm; IVSd- Septal thickness at end diastole, mm; LAd- Left atrial anteroposterior dimension, mm; LVEdD-left ventricular end diastolic dimension, $\mathrm{mm}$; LVEF- left ventricular ejection fraction, $\%$; LVEsD-left ventricular end systolic dimension, mm; LVFS- left ventricular fractional shortening, \%; LVPWd-Posterior wall thickness at end diastole, mm; PA- main pulmonary artery diameter, mm; RVAWdRight ventricular anterior wall thickness at end diastole, mm; RVEdD- Right ventricle outflow diameter at end diastole, $\mathrm{mm}$.

\section{Hosted file}

FIGURE 1.pdf available at https://authorea.com/users/411355/articles/520424-a-study-onnormal-reference-values-of-echocardiographic-chamber-dimensions-in-new-born-infantsfrom-eastern-india-a-pilot-study

\section{Hosted file}

figure 2.pdf available at https://authorea.com/users/411355/articles/520424-a-study-onnormal-reference-values-of-echocardiographic-chamber-dimensions-in-new-born-infantsfrom-eastern-india-a-pilot-study 